\title{
A Comprehensive Overview of Novel Hydroxynitrile Lyases
}

\author{
Asha Kumari ${ }^{1}$, Garima Chauhan ${ }^{1}$, Meghna Arya ${ }^{1}$, and Monica Sharma ${ }^{1}$ \\ ${ }^{1}$ Babasaheb Bhimrao Ambedkar University
}

November 20, 2021

\begin{abstract}
Hydroxynitrile lyases (HNLs) are a heterogeneous family of enzymes that are of particular interest because of their structurally unique categories, a wide range of immobilisation techniques and procedures, and a wide range of sources with varying degrees of enantiopurity and enantioselectivity. Cupin, which contains a new type of HNL from bacteria, the lipocalin superfamily, which has HNLs from millipedes, and the + barrel fold superfamily, which contains HNL from a fern, have all been discovered in recent decades. Their biochemistry has been deciphered, and engineering efforts have been made to boost their productivity, purity, and activity. These remarkable enzymes opened up a new vista in the field of industrial catalysts since they are actively used in the synthesis of crucially important agrochemicals, medicines, physiologically active substances, and chemo-enzymatic follow-up procedures. This review focuses on recent advances, evolutionary history, and recombinant engineering of HNL from the previous decade.
\end{abstract}

\section{A Comprehensive Overview of Novel Hydroxynitrile Lyases}

Asha Kumari, Garima Chauhan, Meghna Arya, Monica Sharma*

Department of Biotechnology, School of Life Sciences, Babasaheb Bhimrao Ambedkar University, Lucknow, Uttar Pradesh, India 226025

*Corresponding author: monashimla@gmail.com, dr.monikas@bbau.,ac.in

Orcid id: 0000-0002-5078-5518

\begin{abstract}
Hydroxynitrile lyases (HNLs) are a heterogeneous family of enzymes that are of particular interest because of their structurally unique categories, a wide range of immobilisation techniques and procedures, and a wide range of sources with varying degrees of enantiopurity and enantioselectivity. Cupin, which contains a new type of HNL from bacteria, the lipocalin superfamily, which has HNLs from millipedes, and the + barrel fold superfamily, which contains HNL from a fern, have all been discovered in recent decades. Their biochemistry has been deciphered, and engineering efforts have been made to boost their productivity, purity, and activity. These remarkable enzymes opened up a new vista in the field of industrial catalysts since they are actively used in the synthesis of crucially important agrochemicals, medicines, physiologically active substances, and chemo-enzymatic follow-up procedures. This review focuses on recent advances, evolutionary history, and recombinant engineering of HNL from the previous decade.
\end{abstract}

Keywords: Hydroxynitrile lyases, Cyanohydrins, Cupin superfamily, Lipocalin superfamily, Mandelonitrile.

\section{Introduction}


Hydroxynitrile lyases catalyse the conversion of cyanohydrins to hydrogen cyanide, aldehydes, or ketones. (Kassim \& Rumbold, 2014; Dadashipour \& Asano, 2011; Sharma et al ., 2005). Plants, arthopods, and bacteria all have HNLs (Hussain et al ., 2012; Wiedner et al ., 2014; Dadashipour et al ., 2015). As a universal defense system against pathogens and herbivores, HNLs are part in the cyanogenic pathway. Cyanogenic glycosides such as amygdalin and prunasin are found in plants and are stored in various tissues, where they are released as hydrogen cyanide when the tissue is damaged (Dadashipour et al ., 2011). Similarly, HNL releases mendalonitrile as an arthopod defence molecule (Dadashipour et al ., 2015). The function of HNLS in bacteria is still unknown. They can synthesize -cyanohydrins and execute the reverse condensation reaction, which results in $\mathrm{C}-\mathrm{C}$ bonds being formed. Cyanohydrins are the building blocks for a wide variety of reactions as well as compounds used in the pharmaceutical, agrochemical, and cosmetic industries (Gruber-Khadjawi et al., 2012, Lanfranchi et al., 2013, Purkarthofer et al., 2007, Winkler et al., 2012). New enzymes with outstanding features are constantly sought after because (R)- and (S)-selective HNLs are used in biocatalysis reactions. Despite the fact that cyanogenic defence mechanisms are used by over 3,000 plant species and that cyanogenic activity varies by family, only a few amino acid sequences have been documented to have proven HNLs activity and are found in common gene and protein sequences (Hickel, 1997; Hernández et al ., 2004; Asanoet al ., 2005; Kassim et al ., 2014).Understanding the amino acid sequence of the enzyme, on the other hand, is critical for recombinant expression and it's engineering. Current HNLs have drawbacks such as low stability (Okrob, 2012), low activity (Hajnal et al . 2013), limited flexibility in expression host selection (Weis. et al ., 2004), and low expression level (Dadashipour et al ., 2015), all of which have an impact on large-scale applications.

\section{Novel sources of Hydroxynitrile lyases}

Despite the fact that several HNLs have been discovered and employed as biocatalysts in industry, scientists have continued to explore for new enzymes in nature (Fig 1). Low-throughput, GC, or HPLC tests made previous screening of these unique enzymes difficult. For direct profiling of HNL activities in plants, Zheng et al . (2021) devised a chromogenic-based high-throughput screening (HTS) technique.

In the same investigation, HNL was isolated from an invading millipede, Chamberlinius hualienensis, a cyanide-emitting arthropod. ChuaHNL was $\mathrm{pH}$ and temperature stable, had broad substrate specificity for aromatic carbonyl aldehydes, and could be used to make enantioselective mandelonitrile. In the production of (R)-mandelonitrile, the enzyme had five times the functional activity of industrial PaHNL. Physicochemical property analysis and logical protein engineering have been used to build industrial biotechnology in a sustainable manner for decades (Purkarthofer et al ., 2007). Millipede HNL was used as a template for isolating successful and promising enzymes, as well as tailoring enzymes through rational protein engineering, both of which are critical for the commercial synthesis of fine compounds and pharmaceuticals (Dadashipoura et al ., 2015). In Parafontaria laminata(PlamHNL) millipedes, another R- specific HNL was found and recombinantly expressed in Pichia pastoris and Escherichia coli (Nuylert et al . 2020). From the seeds of Eriobotrya japonica (loquat), a novel R-selective FAD containing hydroxynitrile lyase was discovered and isolated. The enzyme had a FAD prosthetic group and was exceptionally $\mathrm{pH}$ and temperature stable. The enzyme could degrade both aromatic and aliphatic aldehydes, and it favoured smaller substrates than larger ones (Ueatrongchit et al. 2008). PmHNL, a FAD hydroxynitrile lyase from Prunus mume seed, was isolated, purified, and recombinantly produced in Pichia pastoris, and showed activity over a broad temperature and pH range (Fukuta et al. 2011).

Pratush et al . (2011) discovered heteromeric R-specific HNLs inAbrus precatorius (rosary pea) seeds. Hajnal et al . (2013) identified and successfully expressed a manganese-dependent R- selective hydroxynitrile lyase from the bacterium Granulicella tundricola (GtHNL) in E. coli . In the crystallographic analysis of the HNL crystal, a cupin fold was identified. A conserved barrel domain is found in the cupin protein superfamily (Łyskowski et al. 2012). Enzymes and non-enzymatic storage proteins are both members of the superfamily. GtHNL exhibited neither sequence nor structural relationship to any other HNL and lacked HNL-specific motifs; hence cupins were the first of a new class of HNLs. Through database mining, another highly efficient novel cupin R- selective HNL, Acidobacterium capsulatum (AcHNL), was found. Due to their excellent 
yield on recombinant production inEscherichia coli and increased activity, enantioselectivity, stability, and substrate specificity, these novel cupin HNLs have emerged as a powerful catalyst (Wiedner et al . 2014).

From the young leaves of Nandina domestica Thunb, two distinct R selective HNLs were isolated.The enzymes were different since there was no FAD or carbohydrate. Purified HNLs had molecular weights of 24.9 $\mathrm{kDa}$ and $28.0 \mathrm{kDa}$, respectively, and were designated as NdHNL-S and NdHNL-L. NdHNL-L demonstrated higher functional activity for mandelonitrile production than other plant HNLs, and its amino acid sequence was unique. At $\mathrm{pH} 4.0$ and temperatures below $55^{\circ} \mathrm{C}$, the enzyme catalysed the synthesis of enantioselective (R)-cyanohydrins and showed great activity and stability (Isobe et al. 2018). Although a few new sources of hydroxynitrile lyases have recently been described, HNL is a non-homologous isofunctional enzyme, making identifying new HNL a difficult endeavour (Fig 2).

\section{Evolutionary facet of hydroxynitrile lyases}

Nakano and Asano (2015) designed the INTMSAlign tool to investigate protein engineering and evolution. They utilised the algorithm to make functional proteins, and three S-selective hydroxynitrile lyase (S-HNL)active protein sequences were generated. According to sequencing and biochemical characterization of the generated S-HNLs, neutral mutations increase during the evolution of S-HNLs from a low activity form to a high-activity native form. Overall, the findings showed that software may be used to forecast protein evolution, notably across families like S-HNLs and esterases, in addition to constructing entire sequences.

Because catalytic promiscuity is a valuable but undesired enzyme characteristic, it's difficult to identify reactive promiscuous enzymes in environment. Several primordial enzymes are thought to have been indiscriminate in action because they were at evolutionary branch points of new enzymes. Devamani et al . (2016) put this theory to the test by rebuilding ancestral enzymes at four points when HNLs diverged from esterases. Both enzymes have the identical $\alpha / \beta$-hydrolase-fold, but the reaction forms and mechanisms are different. The ancient enzymes showed more catalytic promiscuity than their successors when tested with esterase and lyase substrates. They discovered that ancient esterases are far more inclined than modern esterases to catalyse a lyase reaction, and ancestral HNLs are more inclined than modern HNLs to catalyse ester hydrolysis. The HNL1 progenitor enzyme was notably promiscuous during the transition from esterase to hydroxynitrile lyases, catalysing both hydrolysis and lyase reactions with a wide range of substrates (Devamani et al. 2016).

In some aspects, the modern rubber tree HNL (HbHNL) differs from HNL1. Catalytic promiscuity has been linked to a diverse active site conformation, a rich biochemical system in the active site interior, and similar transition states. Ester hydrolysis and cyanohydrin cleavage have slightly different transition states and processes. The active site validation alterations can be seen in the x-ray crystallographic structure, such as the substrate binding site residues being more flexible. During ester hydrolysis, serine in the catalytic site acts as a nucleophile, whereas during cyanohydrin cleavage, it behaves as a base. HNL1's ancestral hydroxynitrile lyase has a three-fold higher catalytic promiscuity than modern HbHNL, which is most likely attributable to HNL1's bigger substrate binding site.

The larger binding site allows the flexible substrate p-nitrophenyl acetate (pNPAc), and both of them include aryl groups, however the active catalytic disposition of these groups differs. If the catalytic site is separated into an alcohol binding domain and an aryl binding domain, the aryl group is located in the alcohol binding domain by pNPAc's productive alignment, while the aryl group is located in the aryl binding domain by mandelonitrile's productive alignment. Because a separate substrate orientation is required, a broad active site that can handle both orientations is required, resulting in enhanced catalytic promiscuity. In contrast to HbHNL, the x-ray structure of HNL1 shows a large active site; this difference explains for HNL1's threetime higher ester hydrolysis propensity (Jones et al ., 2020). Higher catalytic promiscuity was also linked to a bigger active site in the alkaline phosphatase family (Barrozo et al ., 2015).

The enhanced thermostability of revived ancestral proteins favours the reconstruction of stabilising substitutions (Trudeau et al ., 2016; Wheeler et al ., 2016; Williams et al , 2006). Because stabilising residues are more likely to be kept in subsequent generations, identifying these residues can be used to precisely determine the ancestral residue. Unstable residues, on the other hand, are more prone to loss in descen- 
dants and hence disregarded when determining ancestral series. As proteins diverge, destabilising residues are altered, resulting in stabilizing residues to counter the impact of substitutions that improve additional properties (Tokuriki \& Tawfik, 2009). As a result of this propensity, any unstable residues discovered in ancestral proteins are unlikely to be conserved in modern proteins, and hence cannot be accurately predicted in a reconstructed antecedent. As a result, ancestral proteins like HNL1 might be rebuilt, and they could be more stable than their real predecessor (Joneset al . 2020).

The structural and functional properties of HNLs are distinct. The catalytic mechanisms of the HbHNL and AtHNL, which have opposite enantioselectivity against mandelonitrile, have been determined to be unique. To determine out how this change happened, they built ancestor-like synthetic active-sites in each subdomain. HbHNL kept (S)-selectivity, whereas AtHNL' with identical active site residues retained (R)selectivity. (A unique mechanism is suggested by (S)-selective composite active-site mutants lacking Lys236 and Thr11, which are required for the conventional (S)-HNL pathway. This new mechanism, which isn't found in modern enzymes, was discovered by simulation. As a result, the last common ancestor of both HbHNL and AtHNL is likely to have used an unknown mechanism rather than the AtHNL-like mechanism. With the same catalytic residues, several pathways are possible, and residues present on the exterior of the active site have a significant impact on mechanism and enantioselectivity (Joneset al . 2017).

Hydroxynitrile lyases are non-homologous isofunctional enzymes that have evolved through convergent evolution (Omelchenko et al . 2010). A unique HNL enzyme was isolated from the fern Davallia tyermannii, and crystallographic analyses indicated that it has a Beta v1-like protein structure, a novel catalytic core, and HNL activity. To find expressed genes, researchers employed normalised transcriptomes from $D$. tyermanii leaves and croziers. Proteins from active tissues were separated using anion exchange chromatography, followed by a native PAGE coupled to HCN detection assay. After isolating the enzymatically active fractions, the protein was digested with trypsin and analysed using LC-MS. The nucleotide transcriptome database of D. tyermannii was compared to peptide data. In E. coli, the sequences were recombinantly expressed and HNL activity was evaluated; isotig02643 had the best chance of being an HNL-active protein. The translated nucleotide transcriptome database revealed three additional remarkably similar sequences to the reported HNL; these gene sequences were amplified from genomic DNA by PCR and their nucleotides validated. After E.coli recombinant synthesis, the HNL activity assay revealed activity of all three isoenzymes, and four proteins were encoded. The uniqueness of DtHNL was investigated using BLAST. The derived DtHNL is distinct from other HNL groups, as evidenced by its low identity and sequence coverage (Lanfranchi et al ., 2017).

Padhi et al. (2010) used only two amino acid modifications to turn a mutant variant of a plant esterase, SABP2, into a hydroxynitrile lyase. The capacity of SABP2-G12T-M239K to catalyse ester hydrolysis was lost, while it gained the ability to catalyse cyanide release from mandelonitrile. This modification also accelerated the reversible reaction, resulting in the formation of mandelonitrile with low enantioselectivity. Mandelontrile lysis has a specificity constant 13,000 times higher than the unaided reaction and is only 1300-fold lesser efficient than HbHNL.

\section{HNLs recombinant expression and protein engineering}

HNLs have been shown to be quite common in nature, and a variety of microorganisms have been identified, the majority of which have been extensively purified and characterised so far. These enzymes have distinct conformations, molecular weights, $\mathrm{pH}$ optimum conditions, and kinetic properties (Table 1). Saturation mutagenesis and random mutagenesis were employed by Asano and Kawahara (2011) to construct S-selective HNL in Baliospermum montanum. Two created BmHNL double mutants, H103C and N156G, showed improved enantioselectivity and specificity for (S)-mandelonitrile production. Nuylert et al . (2017) evaluated the influence of glycosylation on the hyroxynitrile lyase of Passiflora edulis (PeHNL). The gene was recombinantly expressed in Pichia pastoris and E. coli . In a biphasic system, wild type HNL and recombinant expressed HNL from Pichia pastoris outperformed HNL from E. coli and the deglycosylated N105Q mutant of Pichia pastoris expressed HNL in terms of $\mathrm{pH}$, thermal stability, and solvent tolerance. The results demonstrated that the stability profile of HNL may be enhanced by employing a better enzyme 
expression system and N-glycosylation (Nuylert et al .2017a).

According to an X-ray crystallographic investigation of Prunus communis HNL (PcHNL), the side chain of Leucine at 331 position can be fine-tuned to vary the substrate flexibility of large and rigid benzo-ketal aldehyde. This allowed Zheng et al . (2020) to structure-guided redesign HNL by creating an L331A variant that was used in the chemo-enzymatic synthesis of (R)-salmeterol (adrenoreceptor agonist) and obtained $>99 \%$ ee optically pure drug form with $54 \%$ yield. Nuylert et al . (2020) showed thatParafontaria laminata (PlamHNL) millipede HNLs had wider substrate selectivity than other millipede HNLs and may be utilised to make 2-chloromandelonitrile, a critical step in the antithrombotic medication clopidogrel. According to the X-ray crystal structure of PlamHNL, despite having comparable catalytic residues, the binding cavity produced by PlamHNL and Chamberlinius hualienensis is distinct. The PlamHNL-N85Y mutant had 98.2 percent ee purity of 2-chloromandalonitrile and a 91 percent conversion rate, compared to 90 percent ee purity and 76 percent conversion rate for the wild type enzyme (Nuylert et al . 2020).

The HNL coding gene of $P$. edulis f.flavicarpa (PeHNL-Ny) was successfully cloned and expressed to yield recombinant PeHNL-Ny (Asanoet al ., 2005). For KCN and benzaldehyde, the presence of glycan on PeHNL improves catalytic performance without reducing substrate affinity (Nuylert et al ., 2017). Non-glycosylated PeHNL appears to be less stable than glycosylated PeHNL, according to evidence. The structure of PeHNL, as well as a glycosylation site on the protein, improves stability by stabilising the prominent elastic state at the carboxy-terminal. When a 15-amino-acid-residue C-terminal truncated form of PeHNL was made and expressed in E. coli, it produced an enzyme that was not glycosylated. When non-glycosylated and C-terminally truncated mutant PeHNL107 is expressed in P. pastoris, it enhances stability and reusability to the same extent as glycosylated native PeHNL (Nuylert et al ., 2017b).

The ChuaHNL of cyanogenic millipedes exhibited maximum activity for (R)-mandelonitrile synthesis, as well as strong enantioselectivity and stability. HNLs have been found and characterised in eleven cyanogenic millipedes from the Xystodesmidae and Paradoxosatidae families. HNLs are found in all cyanogenic millipedes and according to sequencing research, they may have evolved from a single common ancestor gene. HNL from a different millipede Parafontaria tonominea when expressed inE. coli Shuffle T7, showed strong specific activity for the stability and synthesis of (R)-mandelonitrile throughout a wide temperature and pH range. The presence of disulfide bond gives millipede HNLs their stability. Due to unavailability of the millipedes genome sequences, the ChuaHNL did not exhibit relation to any protein present in the database (Dadashipour et al ., 2015). Yamaguchi et al (2018) isolated an HNL gene from one of these cyanogenic millipedes and sequenced complementary DNA. They designed degenerate primers for amplification of cDNAs of HNL. Five out of eleven HNL cDNA displayed expression in E. coli, and these transgenic cells encoding HNL were employed as enzymes for (R)-mandelonitrile production (Yamaguchiet al ., 2017).

Zhai et al , 2019 investigated the impact of glycosylation on the synthesis of Histidine tagged ChuaHNL in the $P$. pastoris. The codon sequence of the ChuaHNL gene was examined, and it was discovered that the ChuaHNL gene's codons have a high codon bias for P. pastoris, which could be a major bottleneck in the efficient synthesis of His-ChuaHNL in recombinant expression system. As a result, the ChuaHNL gene's codons were altered to P. pastoris-favorable codons. (Sinclair \& Choy, 2002). Hundred codons from ChuaHNL were replaced in this codon optimization, accounting for $61.7 \%$ of all residues. The codon optimization His-SynChuaHNL strain produced 86 times more than His-Ori-ChuaHNL. These findings suggested that the codon's translation competence is a crucial component in increasing ChuaHNL production in the heterologous host P. pastoris (Zhai et al ., 2019).

\section{Reaction mechanism of HNLs}

HNLs catalyse a range of reactions that convert chiral cyanohydrins to hydrocyanic acid, aldehyde, or ketone (Sharma et al . 2005). The reaction mechanism and catalytic residues participating of these R-selective HNLs active sites is shown in Fig 3. Zhu et al . (2015) studied the reaction mechanism of AtHNL using a quantum mechanics/MM approach and proposed two types of response mechanisms. According to the calculations, the cyanohydrin hydroxyl group proton transfer take place first, subsequently the C1-C2 bond 
breaks and rotation of the cyanide ion takes place, and finally, cyanide ion captures a proton from His236 via Ser81. Because the free energy barriers for breaking C1-C2 bonds and protonation of cyanide ions are similar $(12.0 \mathrm{kcal} / \mathrm{mol})$, they both contribute to the rate-limiting step. Path B, with a maximal free energy barrier of 13kcal, deprotonates the cyanohydrin hydroxyl group while simultaneously cleaving the C1-C2 bond. Both paths have similar free energy barriers, therefore either one can be chosen. The reaction's general acid/base is reflected in the catalytic triad formed by His236, Ser81, and Asp208, and the generated cyanide ion is stabilised by Ser81's hydroxyl group and the amne-groups of Ala13 and Phe82 (Zhu et al . 2015).

Motojima et al . (2018) proposed a response mechanism for the lowest HNL in Passiflora edulis (PeHNL). PeHNL is a member of the dimeric $\alpha \beta$ barrel superfamily, which is distinguished by two alpha dimers sandwiching a central $-\beta$ barrel. The $-\mathrm{OH}$ group of $(\mathrm{R})$-mandelonitrile forms $\mathrm{H}$ bonds with His8 and Tyr30 at the binding site, whereas the nitrile group is orientated to the carboxyl group of Glu54 in the docked structure of PeHNL with (R)-mandelonitrile. The catalytic dyad produced by His8-Asn101 is needed for the enzymatic process, according to mutational studies. The hydrogen bond between His-N1 and Asn101-O1 is shorter in the PeHNL-(R)-mandelonitrile complex, increasing the basicity of His8 and allowing it to accept a proton from the $\mathrm{OH}$ group of $(\mathrm{R})$-mandelonitrile. When the cyanide generated by the nitrile group accepts one proton from the protonated His8, HCN is formed. As a result, the His8 in PeHNL's active site behaves as both a general acid and a basic in the process (Motojima et al . 2018).

Motojima et al . (2021) reported a structural analysis of natural ChuaHNL as well as a response mechanism. They looked at the enzyme in the absence of a ligand (1.6), as well as with acetate (1.5), cyanide ions (2.1), thiocyanate (1.55), and iodoacetate inhibitors (1.55). ChuaHNL appears to be a member of the lipocalin superfamily based on its crystal structure. In the docked model of ChuaHNL with its substrate (R)-Mandelonitrile, the hydroxyl group makes hydrogen bonds with Arg38 and Lys117, while the nitrile group creates hydrogen bonds with Arg38 and Tyr103. Lys117 behaves as a base to take a proton from the - $\mathrm{OH}$ group of cyanohydrins, while Arg38 serves as an acid to give a proton to the $\mathrm{CN}^{-}$ion during the bond fission reaction, according to mutational studies. During the cyanohydrin production process, the opposite will happen (Motojima et al . 2021).

Zhao et al . (2014) deduced the reaction mechanism of Hevea brasiliensis HNLs (HbHNL) using ab initio quantum mechanics and molecular dynamics simulations. Ser80, His235, and Asp207 are the catalytic triads of HbHNL's active site, which is buried inside. The substrate can reach the active location through a small route. Catalytic reactions have been discovered to occur in two steps. In the first stage, proton abstraction (double proton transfer) and carbon -carbon bond splitting occurs in quick, coordinated reactions. The second stage involves the production of $\mathrm{HCN}$, which is a rate-determining process. The protonation state of Lys236 has a minimal impact on enzyme function, but it has a significant impact on the catalytic process as a whole. Trp 128 residues act as a gate that adjusts continuously during substrate delivery and HCN release. The Trp128 indole ring acts as a "door" that can be open or closed. As a result of all of this, Helix D's conformation shifts from a -helix to a loop shape. The enzyme releases energy when it binds to a substrate, and product release takes only a little amount of energy $(4.0 \mathrm{kcal}$ mol1 $)$, making the reaction efficient overall (Zhao et al . 2014).

\section{Synthesis of cyanohydrins}

A combination of cassava plant (Manihot esculenta) (S)-specific HNL, nitrile hydratases, and nitrilases was utilised to efficiently produce chiral 2-hydroxycarboxamides and 2-hydroxycarboxylic acids from non-chiral aldehydes/ketones and cyanide (van Rantwijk \& Stolz, 2015). By combining a (R)-specific HNL with nitrilase or nitrile hydratase enzymes, an isofunctional system for generating (R)-2-hydroxycarboxamides and (R)-2hydroxycarboxylic acids was created (Muller et al ., 2021). Recombinant E. coli clones were used to study the synthesis of (R)-mandelic acid and (R)-mandelic acid amide, and strains were generated that expressed a (R)-specific hydroxynitrile lyase from Arabidopsis thaliana as well as the arylacetonitrilase from Pseudomonas fluor. Recombinant strains expressing an acid-tolerant HNL variant and an amide-forming nitrilase variant were produced. In mildly acidic $\mathrm{pH}$ values, whole cell catalysts expressing both the (R)-specific HNL and the wild-type nitrilase converted benzaldehyde and cyanide preferentially to $(\mathrm{R})$-mandelic acid with ee- 
values greater than $90 \%$. The acid and amide producing catalysts transformed chlorinated benzaldehydes to chlorinated mandelic acid/chlorine mandelic acid amides when coupled with cyanide (Muller et al ., 2021).

PlamHNL prom Parafontaria laminata millipedes was cloned and expressed as a host from cDNA. There are 8 Cys residues found in millipede HNLs that generate functional disulfide bonds and are required for HNL folding, activity, and stability (Yamaguchi et al ., 2018). PlamHNL was produced in E. coli and the stereoselectivity for (R)-2-chloromandelonitrile production was enhanced after bacteria were subjected to a computational-directed evolution strategy. Because the enzyme was stable and the gene allowed for continued rational evolution in E. coli, PlamHNL can be employed to produce enantiomerically pure cyanohydrins using structural analyses (Nuylertet al ., 2020)

\section{Future perspective}

Only a few decades ago, genes for $(\mathrm{S})$ and (R)-HNLs were discovered and expressed, and new enzymes are discovered all the time. Several international patent applications typically include manufacturing, improved variants, novel enzymes from various sources (plants, microorganisms, arthropods, etc.), and applications of both (S)- and (R)-HNLs for numerous chemical synthesis. Several enzymes, such as LuHNL, novel and adaptable bacterial HNLS, and synthetic esterases with HNL activity, have become fundamental research targets in addition to patent-protected major enzyme sources. Since then, HNL catalysis has opened up a plethora of new opportunities for a wide range of industrial applications.

\section{Acknowledgement}

We acknowledge the UGC-Junior Research Fellowship given to Ms. Asha Kumari. This research did not receive any specific grant from funding agencies in the public, commercial, or not-for-profit sectors. We also declare that there is no conflict of interest.

\section{References}

1. Alagöz, D., Tükel, S. S.. Yildirim, D. (2014). Purification, immobilization and characterization of $(R$ )-hydroxynitrile lyase from Prunus amygdalus turcomanica seeds and their applicability for synthesis of enantiopure cyanohydrins. Journal of Molecular Catalysis B : Enzymatic , 101, 40-46.

2. Asano, Y., \& Kawahara, N. (2016). A New S -Hydroxynitrile Lyase fromBaliospermum montanum - Its Structure, Molecular Dynamics Simulation, and Improvement by Protein Engineering. Industrial Biotechnology, 12 (2), 91-97.

3. Asano, Y., Tamura, K., Doi, N., Ueatrongchit, T., Kittikun,A. H., and Ohmiya, T . (2005). Screening for new hydroxynitrilase from plants. Bioscience, Biotechnology , and Biochemistry , 69, 2349-2357.

4. Asif, M., \& Bhalla, T. C. (2016) Hydroxynitrile lyase of wild apricot (Prunus armeniaca L.): Purification, characterization and application in synthesis of enantiopure mandelonitrile. Catalysis Letters $146,1118-1127$.

5. Barrozo, A., Duarte, F., Bauer, P., Carvalho, A.T.P., Kamerlin, S.C.L. (2015). Cooperative electrostatic interactions drive functional evolution in the alkaline phosphatase superfamily. Journal of the American Chemical Society, 137, 9061-9076.

6. Dadashipour, M., \& Asano, Y. (2011). Hydroxynitrile lyases: insights into biochemistry, discovery, and engineering. ACS Catalysis , 1, 1121-1149.

7. Dadashipour, M., Ishida, Y., Yamamoto, K., Asano, Y. (2015). Discovery and molecular and biocatalytic properties of hydroxynitrile lyase from an invasive millipede, Chamberlinius hualienensis. Proceedings of the National Academy of Sciences of the United States of America, 112, 10605-10610.

8. Devamani, T., Rauwerdink, A.M., Lunzer, M., Jones, B.J., Mooney, J.L., Tan, M.A.O., Zhang, Z.J., Xu, J.-H., Dean, A.M., Romas, J., Kazlauskas, R.J. (2016). Catalytic Promiscuity of Ancestral Esterases and Hydroxynitrile Lyases. Journal of the American Chemical Society, 138(3), 1046-1056.

9. Fukuta, Y., Nanda, S., Kato, Y., Yurimoto, H., Sakai, Y., Komeda, H., Asano, Y. (2011). Characterization of a new (R)-hydroxynitrile lyase from the Japanese apricot Prunus mume and cDNA cloning and secretory expression of one of the isozymes in Pichia pastoris.Bioscience, Biotechnology, and 
Biochemistry,75(2), 214-220.

10. Gruber-Khadjawi, M., Fechter, M. H., Griengl, H. (2012) Enzyme Catalysis in Organic Synthesis, 947-990.

11. Hajnal, I., Lyskowski, A., Hanefeld, U., Gruber, K., Schwab, H., Steiner, K. (2013). Biochemical and structural characterization of a novel bacterial manganese-dependent hydroxynitrile lyase. FEBS Journal , 280(22), 5815-5828.

12. Hernandez, L., Luna, H., Ruuz-Terán, F., Vázquez, A. (2004). Screening for hydroxynitrile lyase activity in crude preparations of some edible plants. Journal of Molecular Catalysis B : Enzymatic, 30, 105108.

13. Hickel, A., Heinrich, G., Schwab, H., Griengl, H. (1997) Screening for hydroxynitrile lyases in plants.Biotechnology Techniques, 11, 55-58.

14. Hussain, Z., Wiedner, R., Steiner, K., Hajek, T., Avi, M., Hecher, B., Sessitsch, A., Schwab, H. (2012) Characterization of Two Bacterial Hydroxynitrile Lyases with High Similarity to Cupin Superfamily Proteins. Applied and Environmental Microbiology , 78. 2053-2055.

15. Isobe, K., Kitagawa, A., Kanamori, K., Kashiwagi, N., Matsui, D., Yamaguchi, T., Fuhshuku, K-I., Semba, H., Asano, Y. (2018). Characterization of a novel hydroxynitrile lyase from Nandina domestica Thunb. Bioscience Biotechnology and Biochemistry , 82(10), 1-10.

16. Jones, B.J., Evans III, R.L., Mylrea, N.J., Chaudhury, D., Luo, C., Guan, B., Pierce C.T., Gordon, W.R., Wilmot, C.M., Kazlauskas R.J. (2020). Larger active site in an ancestral hydroxynitrile lyase increases catalytically promiscuous esterase activity. PLoS ONE , 15(6), e0235341.

17. Jones, B.J., Bata, Z., Kazlauskas, R. J. (2017). Identical active sites in hydroxynitrile lyases show opposite enantioselectivity and reveal possible ancestral mechanism. ACS Catalysis , 7(6), 4221-4229.

18. Kassim, M. A., Sooklal, S. A., Archer, R., Rumbold, K. (2014). Screening for hydroxynitrile lyase activity in non-commercialised plants. South African Journal of Botany 93, 9-13.

19. Lanfranchi, E., Pavkov-Keller, T., Koehler, E.M., Diepold, M., Steiner, K., Darnhofer, B., Hartler, J., Van Den Bergh, T., Joosten, H. J., Gruber-Khadjawi, M., Thallinger, G. G., Birner-Gruenberger, R. Gruber. K., Winkler, M., Glieder, A. (2017). Enzyme discovery beyond homology: a unique hydroxynitrile lyase in the Bet v1 superfamily.Scientific Reports , 3(7), 46738.

20. Lanfranchi, E., Steiner, K., Glieder, A., Hajnal, I., Sheldon, R.A., van Pelt, S., Winkler, M. (2013). Mini-review: recent developments in hydroxynitrile lyases for industrial biotechnology.Recent Patents on Biotechnology, 7(3), 197-206.

21. Łyskowski, A., Steiner, K., Hajnal, I., Steinkellner, G., Schwab, H., Gruber, K. (2012). Crystallization of a novel metal-containing cupin from Acidobacterium sp. and preliminary diffraction data analysis. Acta crystallographica. Section F, Structural Biology and Crystallization Communications , 68(4):451-454.

22. Motojima, F., Izumi, A., Nuylert, A., Zhai, Z., Dadashipour, M., Sayaka Shichida, S., Yamaguchi, T., Nakano, S., Asano, Y. (2021). R-hydroxynitrile lyase from the cyanogenic millipede, Chamberlinius hualienensis -A new entry to the carrier protein family Lipocalines. FEBS Journal, 288(5):1679-1695.

23. Motojima, F., Nuylert, A., Asano, Y. (2018). The crystal structure and catalytic mechanism of hydroxynitrile lyase from passion fruit,Passiflora edulis . FEBS Journal, 285(2), 313-324.

24. Müller, E., Sosedov, O., Groning, J.A.D., Stolz, A. (2021). Synthesis of $(R)$-mandelic acid and $(R$ )-mandelic acid amide by recombinant $E$. coli strains expressing a $(R)$-specific oxynitrilase and an arylacetonitrilase. Biotechnology Letter, 43, 287-296.

25. Nakano, S., \& Asano, Y. (2015). Protein evolution analysis of S-hydroxynitrile lyase by complete sequence design utilizing the INTMSAlign software. Scientific Reports, 5, 8193.

26. Nuylert, A., Ishida, Y., Asano, Y. (2017a). Effect of Glycosylation on the Biocatalytic Properties of Hydroxynitrile Lyase from the Passion Fruit, Passiflora edulis : A Comparison of Natural and Recombinant Enzymes. Chem BioChem , 18 (3), 257-265.

27. Nuylert, A., Ishida, Y., Asano, Y. (2017b). Stabilization of Hydroxynitrile Lyases from Two Variants of Passion Fruit, Passiflora edulis Sims and Passiflora edulis Forma flavicarpa, by C-Terminal Truncation. ChemBioChem , 18, 257-265. 
28. Nuylert, A., Nakabayashi, M., Yamaguchi, T., Asano, Y. (2020). Discovery and Structural Analysis to Improve the Enantioselectivity of Hydroxynitrile Lyase from Parafontaria laminata Millipedes for ( $R$ )-2-Chloromandelonitrile Synthesis. ACS Omega , 5(43),7896-27908.

29. Okrob, D., Metzner, J., Wiechert, W., Gruber, K., Pohl, M. (2012). Tailoring a stabilized variant of hydroxynitrile lyase from Arabidopsis thaliana. ChemBioChem , 13, 797-802.

30. Omelchenko, M. V., Galperin, M.Y., Wolf, Y. I., Koonin, E.V. (2010). Non-homologous isofunctional enzymes: a systematic analysis of alternative solutions in enzyme evolution. Biology Direct , 5,31 .

31. Padhi, S.K., Fujii, R., Legatt, G.A., Fossum, S.L., Berchtold, R., Kazlauskas R.J. (2010). Switching from an Esterase to a Hydroxynitrile Lyase Mechanism Requires Only Two Amino Acid Substitutions. Chemistry \& Biology, 17(8), 863-871.

32. Pratush, A., Sharma, M., Seth, A., Bhalla, T.C. (2011). Seeds of rosary pea, Abrus precatorius: A novel source of hydroxynitrile lyase.Journal of Biochemical Technology , 3, 274-279

33. Purkarthofer, T., Skranc, W., Schuster, C., Griengl, H. (2007) Potential and capabilities of hydroxynitrile lyases as biocatalysts in the chemical industry. Applied Microbiology and Biotechnology , 76, 309-320.

34. Sharma, M., Sharma, N. N., Bhalla, T.C. (2005)nHydroxynitrile lyases: At the interface of biology and chemistry.Enzyme and Microbial Technology , 37, 279-294.

35. Sinclair, G., \& Choy, F.Y. (2002). Synonymous codon usage bias and the expression of human glucocerebrosidase in the methylotrophic yeast, Pichia pastoris . Protein Expression and Purification, 26, 96-105.

36. Tokuriki, N., \& Tawfik, D. S. (2009) Stability effects of mutations and protein evolvability. Current Opinion in Structural Biology, 19, 596-604.

37. Trudeau, D. L., Kaltenbach, M., Tawfik, D. S. (2016). On the potential origins of the high stability of reconstructed ancestral proteins. Molecular Biology and Evolution , 33, 2633-2641.

38. Ueatrongchit, T., Kayo, A., Komeda, H., Asano, Y., H-Kittikun, A. (2008). Purification and characterization of a novel (R)-hydroxynitrile lyase from Eriobotrya japonica (Loquat).Bioscience, Biotechnology , and Biochemistry , 72(6), 1513-1522.

39. van Rantwijk, F., \& Stolz, A. (2015) Enzymatic cascade synthesis of (S)-2-hydroxycarboxylic amides and acids: cascade reactions employing a hydroxynitrile lyase, nitrile-converting enzymes and an amidase. Journal of Molecular Catalysis B : Enzymatic, 114, 25-30.

40. Wajant, H., Forster, S., Selmar, D., Effenberger, F., Pfizenmaier, K. (1995) Purification and characterization of a novel $(R)$-mandelonitrile lyase from the fern Phlebodium aureum . Plant Physiology, 109, 1231-1238.

41. Weis, R., Poechlauer, P., Bona, R., Skranc, W., Luiten, R., Wubbolts, M., Schwab, H., Glieder, A. (2004). Biocatalytic conversion of unnatural substrates by recombinant almond R-HNL isoenzyme 5.Journal of Molecular Catalysis B : Enzymatic , 29, 211-218.

42. Wheeler, L. C., Lim, S. A., Marqusee, S., Harms, M. J. (2016). The thermostability and specificity of ancient proteins. Current Opinion in Structural Biology, 38, 37-43.

43. Wiedner, R., Gruber-Khadjawi M., Schwab, H., Steiner K. (2014). Discovery of a novel (R)-selective bacterial hydroxynitrile lyase fromAcidobacterium capsulatum . Computational and Structural Biotechnology Journal , 10 (16), 58-62.

44. Williams, P. D,, Pollock, D. D., Blackburne, B. P., Goldstein, R. A. (2006) Assessing the accuracy of ancestral protein reconstruction methods. PLOS Computational Biology , 2(6), e69.

45. Winkler, M., Glieder, A., Steiner, K. (2012). In Comprehensive Chirality (eds Carreira, E. M. \& Yamamoto, H. 7, 350-371

46. Yamaguchi, T., Kuwahara, Y., Asano, Y. (2017). A novel cytochrome P450, CYP3201B1, is involved in (R)-mandelonitrile biosynthesis in a cyanogenic millipede. FEBS Open Bio , 335-347.

47. Yamaguchi, T., Nuylert, A., Ina, A., Tanabe, T., Asano, Y. (2018) Hydroxynitrile lyases from cyanogenic millipedes: molecular cloning, heterologous expression, and whole-cell biocatalysis for the production of (R)-mandelonitrile. Scientific Reports , 8, 3051.

48. Zhai, Z., Nuylert, A., Isobe, K., Asano, Y. (2019). Effects of codon optimization and glycosylation on 
the high-level production of hydroxynitrile lyase from Chamberlinius hualienensis in Pichia pastoris. Journal of Industrial Microbiology and Biotechnology , 46(7), 887-898.

49. Zhao, Y., Chen, N., Mo, Y., Cao, Z. (2014). A full picture of enzymatic catalysis by hydroxynitrile lyases from Hevea brasiliensis : protonation dependent reaction steps and residue-gated movement of the substrate and the product. Physical Chemistry Chemical Physics , 16, 26864-26875

50. Zheng, Y-C., Ding, L-Y., Jia, Q., Lin, Z., Hong, R., Yu, H-L., Xu, J-H., (2021). A High-Throughput Screening Method for the Directed Evolution of Hydroxynitrile Lyase towards Cyanohydrin Synthesis. ChemBioChem, 22 (6), 996-1000.

51. Zheng. Y.C., Li, F-L., Lin, Z., Lin, G-Q., Hong, R., Yu, H-L., Xu, J-H. (2020). Structure-Guided Tuning of a Hydroxynitrile Lyase to Accept Rigid Pharmaco Aldehydes. ACS Catalysis, 10, 5757-5763.

52. Zhu, W., Liu, Y., Zhang, R. A. (2014). QM/MM study of the reaction mechanism of (R)-hydroxynitrile lyases from Arabidopsis thaliana (AtHNL). Proteins , 83(1), 66-77.

Table 1. The biochemical and kinetic properties of novel hydroxynitrile lyase sources

\begin{tabular}{|c|c|c|c|c|c|c|c|c|c|}
\hline Source & Enantiosele & ecféchtyriques & $\begin{array}{l}\text { Subunit } \\
\text { com- } \\
\text { posi- } \\
\text { tion }\end{array}$ & $\begin{array}{l}\text { Molecular } \\
\text { weight }\end{array}$ & $\begin{array}{l}\text { Optimum } \\
\text { Tem- } \\
\text { pera- } \\
\text { ture } \\
\text { and } \\
\text { pH }\end{array}$ & $\begin{array}{l}\text { Kinetic } \\
\text { pa- } \\
\text { rame- } \\
\text { ter }\end{array}$ & $\begin{array}{l}\text { Specific } \\
\text { activ- } \\
\text { ity }\end{array}$ & $\begin{array}{l}\text { Purification } \\
\text { Fold }\end{array}$ & $\mathbf{n}_{\mathbf{R e}}$ \\
\hline $\begin{array}{l}\text { Abrus pre- } \\
\text { catorius } \\
\text { (rosary } \\
\text { pea) Seeds }\end{array}$ & $\begin{array}{l}\mathrm{R}- \\
\text { selective }\end{array}$ & $\begin{array}{l}\text { Ion } \\
\text { exchange } \\
\text { chro- } \\
\text { matogra- } \\
\text { phy Gel } \\
\text { filtration } \\
\text { chromatograp }\end{array}$ & Heteromer & $\begin{array}{l}\text { Native } \\
\text { molecular } \\
\text { mass } 205 \\
\text { kDa Sub- } \\
\text { units: } 42.0 \\
\text { kDa and } \\
36.5 \mathrm{kDa}\end{array}$ & $\begin{array}{l}30^{\circ} \mathrm{C} \text { with } \\
0.1 \mathrm{M} \\
\text { sodium } \\
\text { citrate } \\
\text { buffer ( } \mathrm{pH} \\
5) .\end{array}$ & $\begin{array}{l}\mathrm{K}_{\mathrm{m}}= \\
13 \mathrm{mM} \\
\mathrm{V}_{\max }= \\
625 \mathrm{U} / \mathrm{mg}\end{array}$ & $577 \mathrm{Umg}^{-1}$ & 9 fold & \\
\hline \multicolumn{2}{|c|}{$\begin{array}{l}\text { Acidobacteriufh-selective } \\
\text { capsula- } \\
\text { tum } \\
\text { (expressed } \\
\text { in E. coli) }\end{array}$} & $\begin{array}{l}\text { Anion } \\
\text { exchange } \\
\text { chro- } \\
\text { matogra- } \\
\text { phy Size } \\
\text { exclusion } \\
\text { chromatograp }\end{array}$ & \multicolumn{2}{|c|}{ Homotetrame $4.25 \mathrm{kDa}$} & $\begin{array}{l}\mathrm{pH} 5.5 \\
25^{\circ} \mathrm{C}\end{array}$ & NA & $\begin{array}{l}0.69 \\
\mathrm{Umg}^{-1}\end{array}$ & $2 \%$ yield & \\
\hline & S-selective & $\begin{array}{l}\text { Ammonium } \\
\text { sulfate }\end{array}$ & Homodimer & $\begin{array}{l}60-65 \mathrm{kDa} \\
\text { non-FAD }\end{array}$ & $\begin{array}{l}\mathrm{pH} 5,20 \\
{ }^{\circ} \mathrm{C}\end{array}$ & $\mathrm{k}_{\mathrm{cat}}=98 / \mathrm{s}$ & $178 \mathrm{Umg}^{-1}$ & & \\
\hline \multicolumn{2}{|c|}{ Baliospermum } & $\begin{array}{l}\text { fractiona- } \\
\text { tion }\end{array}$ & & $\begin{array}{l}\text { enzyme } \\
\text { (gel }\end{array}$ & & & & & \\
\hline \multirow{2}{*}{\multicolumn{2}{|c|}{$\begin{array}{l}\text { mon- } \\
\text { tanum }\end{array}$}} & column & & filtration) & & & & & \\
\hline & & chromatograp & phies & $29.5 \mathrm{kDa}$ & & & & & \\
\hline Leaves & & & & & & & & & \\
\hline
\end{tabular}




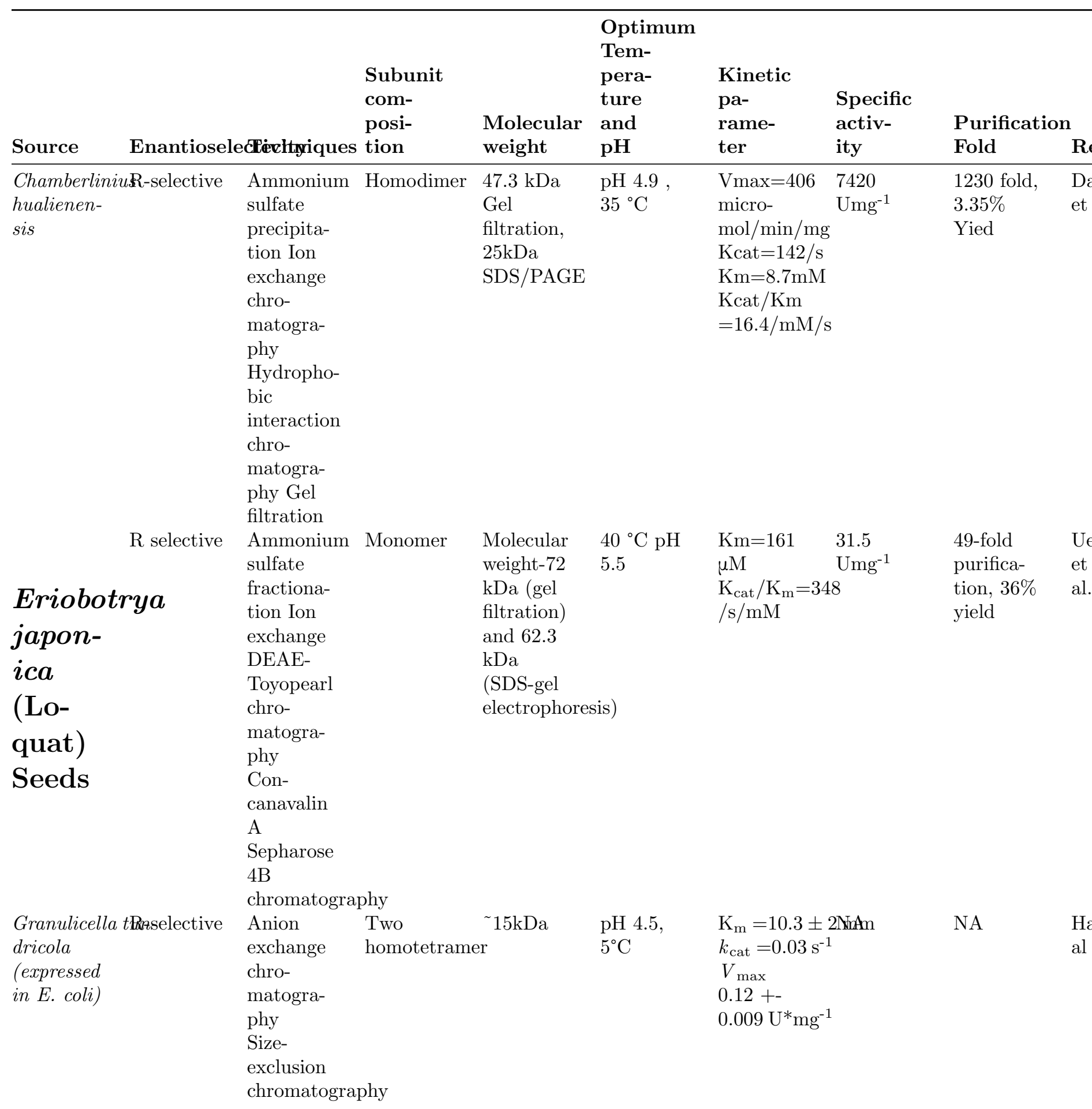




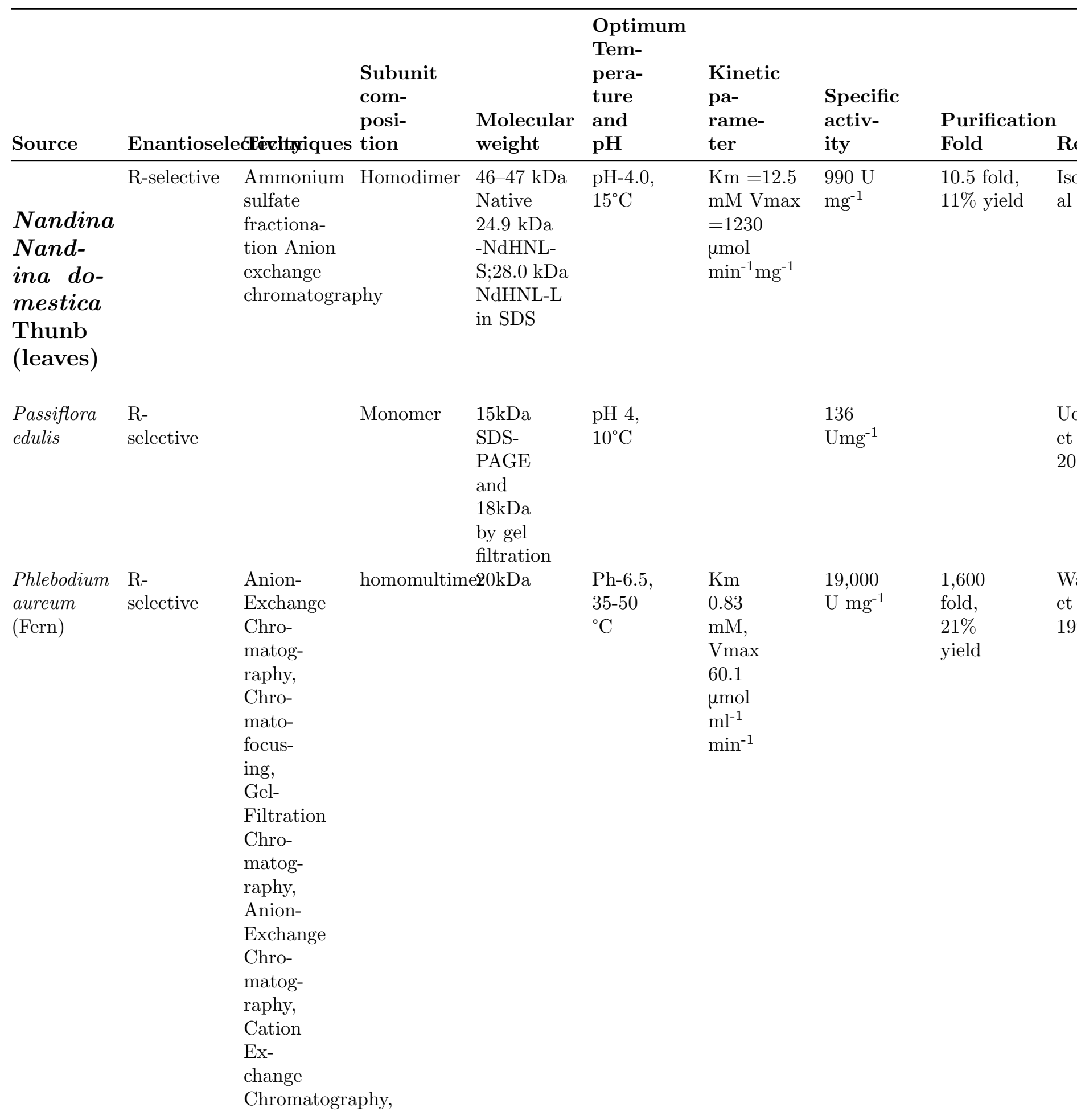




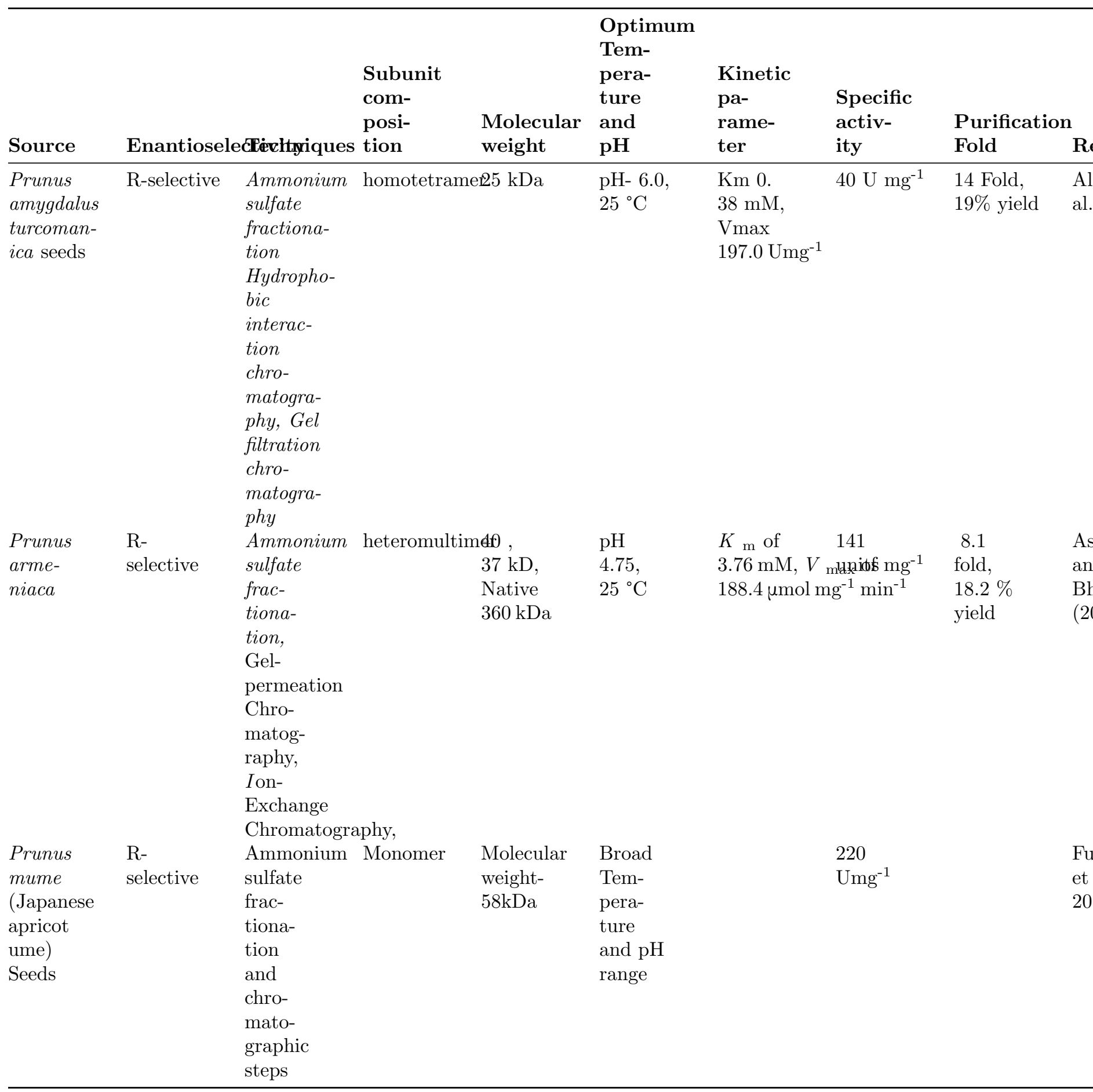

\section{Hosted file}

Manuscript Table.docx available at https://authorea.com/users/447091/articles/546167-acomprehensive-overview-of-novel-hydroxynitrile-lyases

\section{Hosted file}


Manuscript Figure.docx available at https://authorea.com/users/447091/articles/546167-acomprehensive-overview-of-novel-hydroxynitrile-lyases 